\title{
Hablantes de herencia en un programa de inmersión: Diseño de herramientas de evaluación inicial
}

Marta Vega DíEZ

University Studies Abroad Consortium-Alicante

marta.devega@usac.edu

ASUNCIÓN MARTíneZ ARBELAIZ

University Studies Abroad Consortium

asuncion.martinez@usac.edu

Resumen: La creciente presencia de hablantes de herencia en los programas de español como segunda lengua de España nos ha hecho percatarnos de las necesidades de este tipo de alumno. Por ello, resumimos las investigaciones y reflexiones que se han llevado a cabo en diferentes universidades estadounidenses sobre los hablantes de herencia en clases universitarias, enfocándonos en las pruebas de clasificación. En primer lugar, definimos el perfil del hablante de herencia, haciendo énfasis en sus fortalezas y debilidades lingüísticas y destacando lo que lo distingue de un alumno de español como segunda lengua. A continuación, describimos los intentos que se han hecho en diferentes universidades con una presencia importante de alumnado hispano por diseñar, pilotar y administrar exámenes de clasificación exclusivos para este alumnado. Concluimos reflexionado sobre qué tipo de prueba de clasificación ofrecería la correcta ubicación de los hablantes de herencia en los programas de inmersión.

Palabras clave: hablantes de herencia, examen de clasificación, programas de inmersión, educación superior.

\section{Heritage speakers in a study abroad program: Designing a preliminary placement exam}

Abstract: The growing presence of heritage speakers in our study abroad programs in Spain has made us aware of the specific needs of this type of student. First, we summarize research and reflections that have taken place in different U.S. universities regarding heritage speakers, focusing on the placement exam. Then, we define the profile of the heritage speaker, emphasizing linguistic strengths and weaknesses and what distinguishes him/her from an L2 learner. Finally, we describe the attempts that different US universities with large numbers of Spanish-speaking students have made to design, pilot and administer placement exams for these students. We conclude by pondering on what type of placement exam may be the most adequate for heritage speakers when abroad.

Key words: heritage speakers, placement exam, study abroad, higher education.

\section{Contextualización: español para hablantes de herencia en España}

En los últimos años, los que trabajamos en programas de español para universitarios en España o lo que en inglés se denomina study abroad, nos encontramos con una creciente presencia de alumnos hablantes español como lengua de herencia provenientes 
de universidades norteamericanas. En este artículo adoptamos los términos "lengua de herencia" (LH) y "hablante de herencia" (HH), ampliamente usados en la tradición académica estadounidense (Montrul, 2016; Ortega, 2020; Polinsky y Scontras, 2019; 2020; Potowski, 2018), a pesar de que no son de uso común, al menos hasta ahora, en la tradición filológica o educativa española (Cf. Burgo, 2018, López-García, 2017 o Marqués Pascual, en prensa, como excepciones). Wiley (2001:34) comenta que otras posibles etiquetas, como "lengua indígena", "lengua minoritaria/minorizada" o "lengua comunitaria" tampoco están exentas de problemas. Este autor señala que la primera denominación tiene cierto estigma, mientras que la segunda, muy utilizada en publicaciones sobre educación y sociolingüística de ámbito europeo, provoca aversión en Estados Unidos. La tercera denominación es de uso más común en el Reino Unido y Australia.

Pero, ¿qué entendemos por un hablante de herencia? La definición de Valdés (2001:38), canónica en el campo de la educación, describe a un HH como «un individuo que se ha criado en un hogar donde se habla una lengua distinta al inglés, que habla o al menos comprende ese idioma, y que es, hasta cierto punto, bilingüe en esa lengua y el inglés». Ortega (2020) realiza una cuidadosa revisión de la caracterización del HH desde los campos de la sociolingüística, la educación y la lingüística, y reseña rasgos como un bilingüismo "desequilibrado" (unbalanced bilingualism) con mayor dominancia de la lengua mayoritaria, la escasa alfabetización en la lengua de la familia o la relación jerárquica entre ambas lenguas.

Como es sabido, una de las lenguas históricamente habladas en los hogares estadounidenses es el español y su presencia parece aumentar en cada censo que se realiza (Lacorte y Suárez García, 2014). Para dar respuesta a esta realidad social, los departamentos de español de las universidades estadounidenses llevan años diseñando programas de estudios específicos y adecuados a las necesidades de los HHs de español, sobre todo en aquellos estados con mayor presencia hispana, como pueden ser California, Nuevo México o Texas. Carreira y Kagan (2018:160) afirman que el panorama del estudio de las LHs en la enseñanza superior es más optimista que en primaria o secundaria. Sin embargo, estas autoras también señalan el descenso de matrícula en lenguas en general y la falta de profesorado cualificado en el ámbito universitario. Si bien idealmente se debería atender a las necesidades de los HHs desde la educación primaria, en muchos casos los alumnos que llegan a la universidad nunca han recibido instrucción en la lengua que utilizan en casa en mayor o menor medida. En cuanto a otros lugares distintos de los Estados Unidos, como son países europeos, Canadá y Australia, la situación de los $\mathrm{HH}$ de español en el sistema educativo es diversa, dándose casos de integración en el sistema educativo obligatorio, como el ejemplo de Suiza (Sánchez Abchi, 2018), o como una asignatura extraescolar opcional, en el caso de Suecia (Parada, 2018), o ciertos estados alemanes (Ramos MéndezSahlender, 2018). Debido a esta variabilidad en el contacto con la lengua en cuestión, una idea que se repite en todos los estudios dedicados a la enseñanza de LH es que el dominio de dicha lengua es tremendamente heterogéneo (Polinsky y Scontras, 2019; 2020). A menudo, se habla de fenómenos de erosión o desgaste de ciertos aspectos principalmente morfosintácticos. En el caso del español, se suele señalar la vacilación en la asignación de género, de caso, en la elección del modo o en la expresión de la hipótesis (Montrul, 2016). Asimismo, los HHs rara vez han estado expuestos a registros formales de la lengua. Finalmente, otra cuestión importante es que muchos de estos 
alumnos nunca han recibido instrucción en la lectura o escritura de esa lengua, es decir, nunca han tenido contacto con su forma escrita.

Por lo tanto, el primer paso para desarrollar un currículum adecuado a las necesidades de los HHs es comprender la heterogeneidad que presentan en su competencia lingüística debido a las diferencias individuales en los procesos de adquisición. Es cierto que los hablantes de español como segunda lengua también presentan gran variación en cuanto al dominio que demuestran, incluso después de haber completado con éxito los mismos cursos en una universidad dada, pero en el caso de los HHs la disparidad parece ser mayor. Por esta razón, muchas universidades han apostado por una instrucción separada.

Una vez que una universidad y el correspondiente departamento diseñe un currículum de español para HHs, el siguiente paso es decidir qué alumnos están cualificados para completar las clases con éxito. Con objeto de conocer lo que los alumnos HH pueden hacer en la LH, los centros educativos suelen contar con pruebas de evaluación inicial, también llamadas pruebas de nivel, de ubicación, de colocación o de clasificación. Por lo general, los centros educativos cuentan con pruebas propias, ya que, tal y como señala Bordón:

[1]a ventaja de diseñar exámenes de clasificación originales es que serán más coherentes con los niveles existentes en el propio centro y de esta manera, serán, también más efectivos. El proceso de enseñanza-aprendizaje se beneficia, tanto a nivel individual como colectivo, si quienes integran el grupo de clase parten de unos conocimientos (o desconocimientos) similares y tienen unas necesidades comunicativas parecidas. Bordón (2006:81)

Efectivamente, una prueba previa a la instrucción nos permite establecer grupos de alumnos relativamente homogéneos, lo que facilita la labor del profesorado en el aula. Generalmente, dichas pruebas se realizan pensando en un continuum del dominio de la lengua meta como se describe en los niveles del MCER o en los niveles de ACTFL (American Council on the Teaching of Foreign Languages). Las equivalencias entre estos dos sistemas de medir el dominio de la lengua meta, y su relación con el sistema de cursos universitarios estadounidenses, se aborda en Goertler, Kraemer y Schenker (2016). Sin embargo, rara vez se tiene en cuenta en el diseño de estas pruebas que los candidatos tengan un dominio de la lengua que no coincida con los descriptores de estas escalas. Por ello, algunas universidades diseñan sus propias pruebas de clasificación específicas para HHs, como veremos más adelante.

Todas estas innovaciones curriculares tienen un impacto directo en los cursos de lengua que se ofrecen en España o cursos en el extranjero. Como apuntan Shively (2018) y Burgo (2018), los HHs que estudian en el extranjero van en aumento. A veces eligen estudiar en el país de sus ancestros, pero no siempre es el caso. A medida que la presencia de hispanos aumente, también aumentarán los cursos específicos para HHs en España, por ser este el país de habla hispana que más alumnos recibe, seguido de Costa Rica (Institute of International Education, 2019). Sin embargo, la creación de cursos específicos de español para HHs en España no es una tarea fácil y a menudo se discute si las clases separadas es la mejor opción para este tipo de alumnado.

\section{2. ¿Instrucción mixta o separada?}

A pesar de la diversidad dentro de este perfil de estudiantes, existe un consenso en que su conocimiento y habilidades en la LH hacen de ellos un alumnado con unas 
necesidades específicas que difieren de las de los estudiantes de lengua extranjera. Así, el $\mathrm{HH}$, por lo general, ha adquirido la lengua en un contexto informal, mientras que el estudiante de L2 ha seguido una instrucción formal, en la que ha recibido explicaciones explícitas de las reglas gramaticales. Generalmente, esto tiene como resultado una conciencia metalingüística en el estudiante de L2 más desarrollada que en el estudiante HH (Potowski y Lynch, 2014; Bowles, 2018).

Además de la conciencia metalingüística, los HHs tienden a destacar en la realización de tareas, sobre todo si son espontáneas y simulan autenticidad, frente a la mayor destreza de los estudiantes de L2 en actividades dirigidas o controladas, especialmente en el caso de tareas escritas. Carreira y Chik (2018) presentan una síntesis de lo que, en líneas generales, son las fortalezas y debilidades de ambos perfiles en el contexto de aula, recogida en la Tabla 1:

\begin{tabular}{|l|l|}
\hline Aprendiente HH & Aprendiente de L2 \\
\hline $\begin{array}{l}\text { Puede entender y llevar a cabo tareas en la le Sabe mucho sobre la lengua meta. } \\
\text { meta. }\end{array}$ & $\begin{array}{l}\text { Puede manejarse con relativa soltura en activi } \\
\text { simulan la vida real y hacerlo de forma espontâa }\end{array}$ \\
\hline $\begin{array}{l}\text { Puede hablar y entender la lengua } \\
\text { notablemente bien. } \\
\text { aprendizaje) }\end{array}$ & $\begin{array}{l}\text { Puede leer y escribir la lengua meta relativan } \\
\text { bien. }\end{array}$ \\
\hline $\begin{array}{l}\text { Se concentra en el contenido cuando } \\
\text { materiales auténticos en un contexto de aula. }\end{array}$ & $\begin{array}{l}\text { Se concentra en la forma cuando usa mate } \\
\text { auténticos en un contexto formal. }\end{array}$ \\
\hline
\end{tabular}

Tabla 1. Comparación entre los aprendientes HH y los aprendientes de L2

Esta comparación se podría completar con algunos rasgos descritos por Valdés (2001), como son la presencia en el $\mathrm{HH}$ de rasgos estigmatizados, así como de elementos que provienen del contacto con el inglés. De igual modo, la destreza oral implica una conciencia metafórica y la capacidad de comprender y usar el sentido del humor.

Igualmente, otra diferencia con los estudiantes de L2 es que el aprendiente HH está involucrado en procesos cognitivos particulares. Valdés y Parra (2018) señalan que, en un contexto de aula, los procesos de un HH son distintos de aquellos de un L2. Señalan las autoras los siguientes rasgos de los HHs: a) la adquisición de aspectos de su variedad lingüística que han sido adquiridos de forma incompleta; b) la readquisición de aspectos de su variedad lingüística que han tenido un desgaste o involución; c) la adquisición de la variedad estándar de la lengua de herencia como un segundo dialecto y d) el desarrollo de habilidades discursivas, orales y escritas, incluyendo la adquisición de registros y estilos formales. Basándose en evidencias empíricas, Polinsky y Scontras $(2019,2020)$ señalan que el $\mathrm{HH}$ ha recibido una menor cantidad del input, así como una calidad del mismo distinta a la de un nativo, pues generalmente se limita al ámbito familiar y personal. De igual modo, apuntan que el $\mathrm{HH}$ tiene una disponibilidad menor a los recursos de procesamiento de lenguaje. Por último, las necesidades afectivas de los HHs difieren de aquellas de los aprendientes de L2. Mientras que para el primer grupo la lengua supone parte de su identidad, con los consecuentes sentimientos de aceptación o rechazo (Carreira y Chik, 2018; Doerr, 2020; Moreno, 2009; Wilson y Pascual y Cabo, 2019), el segundo grupo no muestra conexiones familiares ni de identidad. 
Por todo ello, hay cada vez más defensores de una instrucción separada y especializada para el $\mathrm{HH}$ o, en el caso de que no sea posible, de una enseñanza diferenciada dentro del contexto de las clases mixtas. No obstante, esta afirmación no está exenta de algunas cuestiones aún por resolver. En primer lugar, el HH que ha aprendido la lengua en un contexto familiar o informal puede tener distintos grados de competencia, dependiendo principalmente de la cantidad y calidad de input que haya recibido. De igual modo, sus experiencias y, por consiguiente, sus actitudes, difieren de unos hablantes a otros en una importante medida. En otras palabras, no hay un $\mathrm{HH}$ igual a otro, $\mathrm{y}$ al igual que un programa educativo diseñado para estudiantes de lengua extranjera no responde a las necesidades de estos alumnos, un curso específico puede no hacerlo si no considera la diversidad presente en este perfil de estudiantes (Beaudrie y Ducar, 2005; Bowles, 2018; Montrul, 2016; Potowski, 2007; Potowski y Lynch, 2014).

En relación con esta cuestión, otro asunto que se ha tratado ampliamente es la evidencia de que la práctica educativa tiene como meta la adquisición de diversos registros de una variedad dialectal estándar -la llamada norma culta- y presta poca o ninguna atención a otras variedades dialectales de menor prestigio. Este hecho resulta una evidencia en las clases diseñadas para estudiantes de L2, pero parece ser también la tendencia en los cursos específicos para HHs (Leeman, 2012). La primera consecuencia es que el hablante nativo pueda sentir que su variedad no es legítima o, incluso, que algunos usos no son correctos (Doerr, 2020; Leeman, 2012; Wilson y Pascual y Cabo, 2019). En este punto, los enfoques más recientes abogan por dar un mayor protagonismo a las experiencias socioculturales de los alumnos (Valdés y Parra, 2018), así como a su variedad dialectal (Beaudrie, 2015) o tener en cuenta sus prácticas propias de hablantes bilingües, como la alternancia de código y el translenguaje (Toribio y Durán, 2018). De esta forma, se ha señalado la efectividad de promover estrategias aditivas o de expansión que favorezcan las transferencias entre las diferentes lenguas y dialectos (Beaudrie, 2015). Así, Valdés $(2001)^{1}$ presenta los posibles escenarios en los que el dialecto del $\mathrm{HH}$ se encuentra con la variedad estándar presentada en el contexto de aula. De forma sintética, se trata de cinco posibilidades: a) elementos lingüísticos que son comunes en ambos dialectos y que se presentan de forma espontánea en el $\mathrm{HH}$; b) elementos que no son producidos en el dialecto no estándar, pero que el $\mathrm{HH}$ podría producir en un contexto formal; c) elementos que el HH podría reconocer y comprender como prestigiados, pero que sería incapaz de producir; d) elementos completamente desconocidos para el $\mathrm{HH}$; e) elementos exclusivos del dialecto no estándar que están altamente estigmatizados en la variedad prestigiada. De igual modo, en los últimos años ha tomado fuerza el denominado enfoque crítico, que tiene por objeto desarrollar las valoraciones y juicios personales sobre la variedad lingüística, de forma que el estudiante pueda ser capaz de determinar la dimensión sociolingüística que tienen las distintas opciones; en palabras de Beaudrie (2015:4) «los estudiantes se empoderarán para determinar qué formas lingüísticas utilizarán con quién y por qué»

En lo referente a la conveniencia de instrucción separada o clases mixtas en un contexto de inmersión, las investigaciones son escasas y no hay evidencias para sostener que se trate de una situación diferente a la de su país de origen. Sin embargo, algunos estudios sugieren los HHs tienen una percepción positiva de su presencia en clases mixtas en contexto de inmersión, si bien los mismos estudios destacan que las ventajas son

\footnotetext{
${ }^{1}$ Valdés hace referencia a la propuesta de Craig (1988), que ella revisa y amplía a los cinco puntos que aquí exponemos.
} 
especialmente notables para los estudiantes de L2 (López García, 2017; Rubin, 2004). Por su parte, también se han estudiado las creencias a este respecto de estudiantes y profesores en contexto de inmersión, concluyendo que ambos grupos perciben que tienen en gran medida necesidades similares, como son el estudio de la gramática, la exposición a textos orales y escritos o la interacción con hablantes locales (Vega-Díez y Martínez Arbelaiz, en prensa).

\section{Hacia una prueba de evaluación inicial específica para HHs}

Nuestro programa de estudios cuenta con una prueba de nivel propia en la que por medio de 85 preguntas de tipo test se intenta tener una visión de la competencia gramatical de los alumnos que vienen a estudiar a un país de habla hispana. Si bien esta prueba está diseñada para estudiantes de español como lengua extranjera, a través de los años ha tenido utilidad como evaluación diagnóstica de los HHs. No obstante, diversos estudios insisten en la importancia de una prueba de evaluación específica en los que obtener unos resultados más adecuados a las necesidades de este perfil de estudiante. Es importante tener en cuenta que los placement tests diseñados para aprendientes de español lengua extranjera pueden tener un impacto negativo en los HHs. Estos pueden ser sancionados al desconocer algunos aspectos de la variedad estándar y terminar considerando que hablan una variedad "incorrecta" o "impura" (Holguín Mendoza, 2018).

Fairclough (2012) propone un modelo para desarrollar exámenes que tenga en cuenta las necesidades de los HHs y propone las siguientes pautas para el diseño de un modelo de evaluación específico:

a) La definición del constructo de nivel de la lengua de herencia.

b) Consideraciones preliminares, como la misión docente, las características del programa y estudiantado y el contenido del curso.

c) El contenido del test (¿qué evaluamos?), lo que los alumnos saben y sus huecos lingüísticos; las habilidades receptivas, productivas y creativas.

d) Diseño del test y tareas: ítems de reconocimiento léxico, dictado, traducción parcial, gramática y producción de verbos; actividades abiertas, como lectura y escritura; escuchar y hablar.

e) Implementación del test: hacer una prueba piloto, analizar los datos obtenidos y solucionar problemas de logística.

Teniendo en cuenta este modelo, nos preguntamos qué forma ha de tener un examen de clasificación dirigido a hablantes de herencia. Creemos que la evaluación inicial de un $\mathrm{HH}$ debería comenzar con una biografía lingüística en la que el individuo describa su propia historia como hablante de esa lengua, así como de la imagen que tiene de su propia competencia. De las distintas propuestas que hay a este respecto, reproducimos la propuesta del Departamento de Hispanic Studies de la universidad de Houston. En su página web (https://www.uh.edu/class/spanish/language-programs/heritage-language/) se puede encontrar las siguientes preguntas que tratan de identificar a los alumnos $\mathrm{HH}$ :

If you answer YES to one or more of the following statements, you need to take the Placement-Credit Exam for Spanish Heritage Learners:

- When I was a child, Spanish was spoken in my home 
- I lived in a Spanish-speaking country for two years or longer

- My first language was Spanish (or both Spanish and English)

- I speak Spanish, or both Spanish and English with family and friends

- My parents and/or grandparents often speak to me in Spanish

La idea de preguntar al alumno por su uso del español fuera del aula y no solo durante la infancia, ha sido adoptada como parte del examen de clasificación en varias universidades. Parece lógico que los alumnos que contesten que usan español en más ámbitos de la vida deberían poder matricularse en cursos más avanzados. Para tener una idea de la competencia lingüística de este alumnado parece necesaria una serie de preguntas tipo test que se autocorrijan. Tal y como propone Fairclough (2012) es importante tener una muestra de su capacidad de escribir. Para ello una combinación de test y de escritura libre parece lo más apropiado. Los ítems del test deberían estar dirigidos a este alumnado. Idealmente, tendrían un índice de dificultad moderado, presentando opciones que los HHs deban discriminar. Un ejemplo de un ítem de nuestro examen online que suele presentar dificultad para los HHs es el siguiente:

(1) ¿Qué vestido te para el concierto de ayer?
a)pusiste
b)pusistes
c)ponistes
d) poniste

Un examen de clasificación para HHs contaría con un número suficiente de ítems de este tipo seguidos de una tarea escrita. Algunas preguntas como sugerencia para la tarea de escritura son las siguientes: ¿Qué objetivos académicos tienes para tu curso en España? ¿Qué te gustaría aprender? En diez o quince líneas explica tus metas para el estudio en el extranjero.

La redacción tanto de los ítems del test como de las tareas de escritura debe ser consensuado entre profesores porque se trata de un trabajo que requiere grandes dosis de creatividad y conocimiento de esta variedad del español (Zabaleta, 2007). Algunas universidades estadounidenses ya han desarrollado exámenes de ubicación específicos para HHs en los que nos podemos inspirar, como veremos en la siguiente sección.

\section{Algunos ejemplos de exámenes de clasificación diseñados para HHs: la Universidad de Illinois en Chicago, la Universidad de Houston y dos universidades del estado de New Mexico}

Prueba de que el campo del español como LH está llegando a su madurez es que contamos con descripciones no solo de los exámenes de clasificación sino de sus correspondientes procesos de pilotaje y de implementación en varias universidades estadounidenses. El volumen 9 número 1 de la revista Heritage Language Journal está dedicado precisamente a este tema. Como bien resume Beaudrie (2012:VII) en la introducción de este número, existe «la necesidad de desarrollar exámenes de clasificación que reflejen los objetivos curriculares y contenidos de cada programa de lengua de herencia y las necesidades lingüísticas y culturales de la población de lengua de herencia local a la que se supone que sirven» (traducción de las autoras). Con objeto de inspirarnos en lo realizado hasta ahora, describimos el proceso de diseño, pilotaje e implementación de tres pruebas de ubicación en cuatro universidades: la universidad de Illinois at Chicago; la Universidad de Houston, New Mexico State University y la Universidad de New Mexico. 
La universidad de Illinois at Chicago cuenta con una de las autoras más activas a la vez que más influyentes en este campo emergente: Kim Potowski. Ella, junto a dos colegas de la misma universidad, emprendieron la tarea de diseñar un examen online que discrimine entre los HHs y los aprendientes de español L2, puesto que, como vimos en la sección 2, tienen características diferenciadas. Potowski, Parada y Morgan-Short (2012) realizaron cinco tests. El primer test es el test C, el cual consta de 4 tipos de ítems: a) Léxico y frases coloquiales, neutros o del dialecto mexicano. b) Cuestiones de ortografía y acentuación. c) Aspectos gramaticales, tales como la diferencia entre gerundio/infinitivo, uso de preposiciones y lo que llaman morfosintaxis coloquial y d) Conocimiento metalingüístico. Según las autoras, solo el primer tipo de ítem sirvió para distinguir entre HHs y los aprendientes de español L2. Los otros cuatro tests, se corresponden fielmente con el contenido de los cursos de esta universidad de Illinois, tanto para HHs como para aprendientes de español L2.

El examen de clasificación de le Universidad de Houston ha sido descrito en varias publicaciones, como Fairclough $(2006,2011,2012)$ y Fairclough, Belpoliti and Bermejo (2010). Al igual que el examen de Illinois, es un examen online y adaptivo; cuenta con un cuestionario biográfico, un ejercicio de traducción, otro de dictado, y varias tareas de gramática, además de una parte creativa oral y escrita. Pero quizá la parte más innovadora y la que lo distingue de otros tests, es la parte que mide el reconocimiento de las piezas léxicas. Esta parte consta de 200 palabras, de las cuales 120 son palabras reales del español organizadas por índices de frecuencia y 80 lo que las autoras llaman "pseudopalabras" o palabras inventadas. Existe una diferencia estadísticamente significativa entre el número de palabras que los $\mathrm{HHs}$ son capaces de reconocer y las que reconocen los alumnos de español L2. Al igual que en el examen de Illinois, todos los tests se corrigen mecánicamente a excepción de las partes que se denominan "creativas", las cuales tienen que corregirse manualmente. Según Potowski et al. (2012:67), en dos o tres minutos es posible analizar la parte escrita y, asimismo, estos resultados proporcionan mucha información de la eficacia del programa al poderse realizar también al final del curso.

Finalmente, contamos con descripciones del examen de clasificación de la Universidad estatal de New Mexico (MacGregor-Mendoza, 2011) y el de la Universidad de New Mexico, recogido en Wilson (2012). MacGregor-Mendoza (2011) hace una revisión del antiguo examen de ubicación de su universidad señalando que se trata del mismo examen para los dos grupos de alumnos; sin embargo, los HHs deben contestar 90 preguntas de opción múltiple, mientras que los alumnos de español como L2 solo tienen que responder a 50. Es decir, que los HHs tienen que contestar 40 preguntas más. Según la autora «[1] os no nativos toman un examen compuesto de las 50 preguntas más básicas $\mathrm{y}$, según el resultado pueden exentarse de cursos hasta el segundo curso intermedio y recibir una recomendación para entrar al curso de gramática. Por contraste, los estudiantes identificados como hablantes de herencia tienen que presentar un examen de casi el doble de ítems, de un contenido más variado y más difícil, y sacar un puntaje mucho mayor para recibir la misma dispensación» (p. 158). Además, la autora hace un análisis crítico de diferentes ítems que resultan ambiguos y que penalizan el conocimiento pragmático frente al puro conocimiento gramatical.

El ítem de examen que reproducimos en (2) ilustra esta idea. La respuesta (c) es pragmáticamente correcta en una situación de comunicación real. Sin embargo, el 
conocimiento del uso más allá de las reglas sobre los pronombres clíticos que repiten los libros de texto hacen que el examen solo acepte (a) como respuesta correcta:

(2) ¿Ellos los conocer a ustedes?
a) nos conoces
c) nos conocemos
b) los conocen
d) los conocemos

Asimismo, el examen presentaba un sesgo hacia los cursos más avanzados, es decir, que la mayoría de los alumnos eran ubicados en estos cursso, en los que las habilidades de lectoescritura ya estaban establecidas. Como vimos en la sección 2, esto no suele ser el caso de los alumnos HHs. Por estas razones, MacGregor-Mendoza propone realizar una prueba independiente para los HHs que incluya ítems auténticos en un orden lógico y en formatos diversos, que presente formatos audiovisuales. Desconocemos si el examen ya está en uso en la Universidad del estado de New Mexico, pero en cualquier caso ningún examen se debe dar como finalizado y como afirma Zabaleta (2007: 689) «el test no debería verse como un producto terminado. Por el contrario, debería ser monitoreado continuamente para aserguranos de que los items del test funcionan como se esperaba» (traducción de las autoras).

En otra universidad del mismo estado, en la Universidad de New Mexico, Wilson (2012) y su equipo pilotaron dos veces un examen de preguntas de opción múltiple. En la primera fase, el examen contó con 104 ítems, los cuales se redujeron a 64 en la segunda fase del pilotaje. Este examen está diseñado para medir diferentes tipos de conocimiento, tales como: a) vocabulario regional, b) vocabulario general, c) pronombres de objeto, d) concordancia de género y número, e) uso de conjugaciones y tiempos, f) comprensión de lectura. El ejemplo (3) muestra un ítem de dicho examen en el que vemos la forma en la que los autores acceden al conocimiento de vocabulario regional por parte de los usuarios. Obviamente, el examen original no incluye la traducción al inglés de las diferentes opciones léxicas:

(3) No me gusta que me piquen los

a. Moyotes (mosquitoes)

b. Barriles (barrels)

c. Alazanes (elks)

Wilson (2012) hace un análisis gráfico del comportamiento de cada uno de los ítems de la prueba eliminándose así 40 ítems que no cumplían con los requisitos estadísticos requeridos. No disponemos de más información sobre cómo quedó el examen de ubicación en su forman final, pero esperamos que haya futuras publicaciones sobre las sucesivas versiones y, sobre todo, sobre su eficacia en la clasificación de los alumnos. En cualquier caso, salvo los ejemplos de ítems concretos que se ofrecen en estas publicaciones, ninguna universidad de las mencionadas ni ninguna otra, ha publicado el examen completo, ya que suele ser información que tiene cierto grado de confidencialidad y es susceptible de ser copiado.

\section{A modos de conclusión}

La presencia de HHs en las aulas de español en las universidades estadounidenses está creciendo y, por consecuencia, también en los programas de inmersión en España y otros países de habla hispana. Por esta razón, es necesario un conocimiento profundo de las necesidades pedagógicas de este alumnado, distinguiéndolos así del alumno de 
español como L2. El primer paso, posiblemente antes de que lleguen a España, es determinar su conocimiento de la lengua meta. Un examen específico, como el que las universidades que hemos mencionado están desarrollando, aportaría mucha luz a la hora de ubicar a estos alumnos en los cursos más apropiados. Dado que los HHs presentan una gran heterogeneidad en cuanto a su conocimiento y dominio del idioma, un buen diagnóstico inicial que nos permita a los profesores conocer las fortalezas y debilidades lingüísticas de este alumnado es el primer paso para el éxito de nuestros cursos.

Creemos que un examen de ubicación para HHs puede contar con dos partes: una de reconocimiento de formas gramaticales que son susceptibles de variación, tales como la elección del subjuntivo, la expresión de la hipótesis, los clíticos o la concordancia de género, entre otros, y una segunda parte en la que obtengamos una pequeña muestra de la producción escrita del alumno en cuestión, puesto que es importante saber su grado de alfabetización. El diseño de ítems de examen con distractores que reflejen las dudas y confusiones que este alumnado pueda tener es la parte más costosa de diseñar. Consideramos que, a diferencia de las pruebas que diseñan las universidades estadounidenses, no es necesario medir el conocimiento del léxico de la variedad regional de EE.UU. Si bien esto puede tener relevancia en Nuevo México o en Texas, para los propósitos de la enseñanza del español en situación de inmersión, este conocimiento pasa a un segundo plano. En conclusión, el diseño de un examen de clasificación específico para HHs empieza a ser una necesidad en los programas de español para universitarios estadounidenses. Hemos dado algunas ideas de lo que se ha realizado hasta ahora en algunas universidades que cuentan con alumnado hispano, así como sugerencias de cómo abordar la tarea del diseño de un examen de clasificación específico en programas de estudios en el extranjero.

\section{Referencias}

BEAUDRIE, S.M. (2012). «Introduction: Developments in Spanish Heritage Language Assessment». Heritage Language Journal, 9 (1), pp. i-xi.

BEAUDRIE, S.M. (2015). "Approaches to language variation: Goals and objectives of the Spanish heritage language syllabus», The Heritage Language Journal, 12 (1), pp. 1-21.

BEAUDRIE, S.M. y DUCAR, C. (2005). «Beginning level university heritage programs: Creating a space for all heritage language learners», Heritage Language Journal, 3 (1), pp. 1-26.

BORdón, T. (2006). La evaluación de la lengua en el marco de E/L2: Bases y procedimientos. Madrid: Arco Libros.

BOWLES, M.A. (2018). «Outcomes of classroom Spanish heritage language instruction». En K. Potowski (ed.), The Routledge handbook of Spanish as a heritage language (pp. 331-344). Nueva York, NY: Routledge.

BuRGO, C. (2018). Clases mixtas: L2 y lengua de herencia. Madrid: Arco Libros.

CARreIRA, M. y CHIK, C.H. (2018). «A primer for heritage and mixed clases». En K. Potowski (ed.), The Routledge handbook of Spanish as a heritage language (pp. 359374). Nueva York, NY: Routledge. 
CARreira, M. y KagAn, O. (2018). «Heritage language education: A proposal for the next 50 years», Foreign Language Annals, 51(1), 152-168.

CRAIG, D.R. (1988). «Creole English and education in Jamaica». En C.B. Paulston (ed.), International handbook of bilingualism and bilingual education (pp. 297-312). Nueva York, NY: Greenwood Press.

DOERR, N.M. (2020). «'Global competence’of minority immigrant students: hierarchy of experience and ideology of global competence in study abroad», Discourse: Studies in the Cultural Politics of Education, 41 (1), pp. 83-97.

FAIRCLOUGH, M.A. (2006). «Language placement exams for heritage speakers of Spanish: Learning from students' mistakes», Foreign Language Annals, 39 (4), pp. 595604.

FAIRCLOUGH, M.A. (2011). «Testing the lexical recognition task with Spanish/English bilinguals in the United States», Language Testing, 28 (2), pp. 273-297.

FAIRCLOUGH, M.A. (2012). «A working model for assessing Spanish heritage language learners' language proficiency through a placement exam», Heritage Language Journal, 9 (1), pp. 121-138.

Fairclough, M.A., Belpoliti, F. y Bermejo, E. (2010). «Developing an electronic placement examination for heritage learners of Spanish: Challenges and payoffs», Hispania, 93 (2), pp. 273-291.

Goertler, S., Kraemer, A. y SchenKer, T. (2016). «Setting Evidence-Based language goals», Foreign Language Annals, 49 (3), pp. 434-454.

Holguín MendozA, C. (2018). «Critical language awareness (CLA) for Spanish heritage language programs: Implementing a complete curriculum». International Multilingual Research Journal, 12 (2), pp. 65-79.

INSTITUTE OF INTERNATIONAL EDUCATION (2019). «Top Ten destinations of US Study Abroad Students», Open Doors Report on International Educational Exchange. Recuperado el 31 de enero 2020 de https://www.iie.org/opendoors

LACORTE, M. y SuÁrez GarcíA, J. (2014). «La enseñanza del español en los Estados Unidos: panorama actual y perspectivas de futuro», Journal of Spanish Language Teaching 1 (2), pp. 129-136.

LEEMAN, J. (2012). "Investigating language ideologies in Spanish as a heritage language». En S. Beaudrie and M. Fairclough (eds.), Spanish as a heritage language in the United States: The state of the field (pp. 43-59). Washington, DC: Georgetown University Press.

LÓPEZ-GARCÍA, M.P. (2017). «La enseñanza del español a hablantes de herencia en inmersión parcial y total: factores eficaces del aprendizaje en aulas heterogéneas», Tejuelo, 26, pp. 113-142.

MACGREGOR-MENDOZA, P. (2011). «Probando, probando... analizando la utilidad de un examen de ubicación para hablantes de español como lengua de herencia». En L.A. Ortiz-López (ed.), Selected Proceedings of the $13^{\text {th }}$ Hispanic Linguistics Symposium (pp. 150-160). Somerville, MA: Cascadilla Proceedings Project. 
MARQues-PASCUAL, L. (en prensa). «Los hablantes de español como lengua de herencia en programas de estudios en el extranjero». En P. Taboada-de-Zúñiga Romero y R. Barros Romero (eds.), Perfiles, factores y contextos en la enseñanza y el aprendizaje de ELE/EL2. Actas del XXIX Congreso Internacional ASELE. Santiago de Compostela: Universidade de Santiago de Compostela.

MONTRUL, S. (2016). The acquisition of heritage languages. Cambridge, UK: Cambridge University Press.

MORENO, K.H. (2009). The study abroad experiences of heritage language learners: Discourses of identity. Tesis doctoral, University of Texas, Austin, TX.

ORTEGA, L. (2020). «The study of heritage language development from a bilingualism and social justice perspective», Language Learning, 70, pp. 15-53.

PARADA, M. (2018). "Chilean Spanish speakers in Sweden: transnationalism, trilingualism, and linguistic systems». En K. Potowski (ed.), The Routledge handbook of Spanish as a heritage language (pp. 517-536). Nueva York, NY: Routledge.

POLINSKY, M. y SCONTRAS, G. (2019). «Understanding heritage languages». Bilingualism: Language and Cognition. 1-17.

POLINSKY, M. y SCONTRAS, G. (2020). «A roadmap for heritage language research», Bilingualism: Language and Cognition, 23 (1), pp. 50-55.

Potowski, K. (2007). Fundamentos de la enseñanza del español a hispanohablantes en los EE. UU. Madrid: Arco Libros.

Potowski, K. (2018). The Routledge handbook of Spanish as a heritage language. Nueva York, NY: Routledge.

PotOWsKi, K. y LYNCH, A. (2014). «Perspectivas sobre la enseñanza del español a los hablantes de herencia en los Estados Unidos», Journal of Spanish Language Teaching, 1 (2), pp. 154-170.

Potowski, K., Parada, M. y Morgan-Short, K. (2012). «Developing an online placement exam for Spanish heritage speakers and L2 students», Heritage Language Journal, 9 (1), pp. 51-76.

RAMOS MÉNDEZ-SAHLENDER, C. (2018). Spanish as a heritage language in Germany. En K. Potowski (ed.), The Routledge handbook of Spanish as a heritage language (pp. 492503). Nueva York, NY: Routledge.

RuBIN, K. (2004). «Going 'Home' to study», International Educator, 13(1), pp. 26-33.

SÁNCHEZ ABCHI, V. (2018). «Spanish as a heritage language in Switzerland». En K. Potowski (ed.), The Routledge handbook of Spanish as a heritage language (pp. 504516). Nueva York, NY: Routledge.

SHIVELY, R.L. (2018). «Spanish Heritage Speakers Studying Abroad». En K. Potowski (ed.), The Routledge handbook of Spanish as a heritage language (pp. 403-419). Nueva York, NY: Routledge.

TORIBIO, A.J. y DURÁN, L. (2018). «Understanding and leveraging Spanish heritage speakers' bilingual practices». En K. Potowski (ed.), The Routledge handbook of Spanish as a heritage language (pp. 284-298). Nueva York, NY: Routledge. 
VALDÉS, G. (2001). «Heritage language students: profiles and possibilities». En J.K. Peyton, D.A. Ranard, y S. McGinnis (eds.), Heritage languages in America: Preserving a national resource (pp. 37-77). McHenry, IL: Center for Applied Linguistics and Delta Systems Co., Inc.

VALDÉS, G. y PARRA, M.L. (2018). «Towards the development of an analytical framework for examining goals and pedagogical approaches in teaching language to heritage speakers». En K. Potowski (ed.), The Routledge Handbook of Spanish as a Heritage Language (pp. 301-330). Nueva York, NY: Routledge.

VegA-Díez, M. y MARTínez Arbelaiz, A. (en prensa). «Creencias del profesorado sobre la enseñanza del español a hablantes de herencia en situación de inmersión» En P. Taboada-de-Zúñiga Romero y R. Barros Romero (Eds.), Perfiles, factores y contextos en la enseñanza y el aprendizaje de ELE/EL2. Actas del XXIX Congreso Internacional ASELE. Santiago de Compostela: Universidade de Santiago de Compostela.

WiLSON, D.V. (2012). «Developing a placement exam for Spanish heritage language learners: Item analysis and learner characteristics», Heritage Language Journal, 9 (1), pp. 27-50.

Wilson, D.V Y PASCUAL Y CABO, D. (2019). «Linguistic diversity and student voice: The case of Spanish as a heritage language», Journal of Spanish Language Teaching, 6 (2), pp. 170-181.

WILEY, T.G. (2001). «On defining heritage languages and their speakers». En J.K. Peyton, D.A. Ranard, y S. McGinnis (eds.), Heritage languages in America: Preserving a national resource (pp. 29-36). McHenry, IL: Center for Applied Linguistics and Delta Systems Co., Inc.

ZABALETA, F. (2007). «Developing a multimedia, computer-based Spanish placement test», CALICO Journal, 23(3), pp. 675-692. 